\title{
Article \\ Granzyme B PET Imaging in Response to In Situ Vaccine Therapy Combined with $\alpha$ PD1 in a Murine Colon Cancer Model
}

\author{
Siddesh V. Hartimath ${ }^{1, *, \dagger}$, Boominathan Ramasamy ${ }^{1,2, \dagger}$, Tan Yun Xuan ${ }^{1}$, Tang Jun Rong ${ }^{1}$, \\ Shivashankar Khanapur ${ }^{1}$, Peter Cheng ${ }^{1}$ (D), You Yi Hwang ${ }^{3}$, Edward G. Robins ${ }^{1,4}$ and Julian L. Goggi ${ }^{1, *}$
}

Citation: Hartimath, S.V.; Ramasamy, B.; Xuan, T.Y.; Rong, T.J.; Khanapur, S.; Cheng, P.; Hwang, Y.Y.; Robins, E.G.; Goggi, J.L. Granzyme B PET Imaging in Response to In Situ Vaccine Therapy Combined with $\alpha \mathrm{PD} 1$ in a Murine Colon Cancer Model. Pharmaceutics 2022, 14, 150. https://doi.org/10.3390/ pharmaceutics 14010150

Academic Editors: Steve J. Archibald and Louis Allott

Received: 12 November 2021

Accepted: 4 January 2022

Published: 8 January 2022

Publisher's Note: MDPI stays neutral with regard to jurisdictional claims in published maps and institutional affiliations.

Copyright: (C) 2022 by the authors. Licensee MDPI, Basel, Switzerland. This article is an open access article distributed under the terms and conditions of the Creative Commons Attribution (CC BY) license (https:// creativecommons.org/licenses/by/ $4.0 /)$.
1 Laboratory of Radiochemistry \& Molecular Imaging (LRMI), Institute of Bioengineering and Bioimaging (IBB), A*STAR Research Entities, Helios, Singapore 138667, Singapore; Boominathan_Ramasamy@ibb.a-star.edu.sg (B.R.); Tan_Yun_Xuan@ibb.a-star.edu.sg (T.Y.X.); Tang_Jun_Rong@ibb.a-star.edu.sg (T.J.R.); Shivashankar@ibb.a-star.edu.sg (S.K.); Peter_Cheng@ibb.a-star.edu.sg (P.C.); edward_robins@ibb.a-star.edu.sg (E.G.R.)

2 Department of Pharmacology, Faculty of Pharmaceutical Sciences, UCSI University, Kuala Lumpur 56000, Malaysia

3 FACS facility, Singapore Immunology Network (SIgN), A*STAR Research Entities, Immunos, Singapore 138665, Singapore; leon_hwang@immunol.a-star.edu.sg

4 Clinical Imaging Research Centre (CIRC), Yong Loo Lin School of Medicine, National University of Singapore, Singapore 117599, Singapore

* Correspondence: s_hartimath@ibb.a-star.edu.sg (S.V.H.); Julian_Goggi@ibb.a-star.edu.sg (J.L.G.)

+ These authors contributed equally to this work.

\begin{abstract}
Immune checkpoint inhibitors (ICIs) block checkpoint receptors that tumours use for immune evasion, allowing immune cells to target and destroy cancer cells. Despite rapid advancements in immunotherapy, durable response rates to ICIs remains low. To address this, combination clinical trials are underway assessing whether adjuvants can enhance responsiveness by increasing tumour immunogenicity. CpG-oligodeoxynucleotides (CpG-ODN) are synthetic DNA fragments containing an unmethylated cysteine-guanosine motif that stimulate the innate and adaptive immune systems by engaging Toll-like receptor 9 (TLR9) present on the plasmacytoid dendritic cells (pDCs) and B cells. Here, we have assessed the ability of AlF-mNOTA-GZP, a peptide tracer targeting granzyme B, to serve as a PET imaging biomarker in response to CPG-ODN 1585 in situ vaccine therapy delivered intratumourally (IT) or intraperitoneally (IP) either as monotherapy or in combination with $\alpha$ PD1. $\left[{ }^{18} \mathrm{~F}\right]$ AlF-mNOTA-GZP was able to differentiate treatment responders from non-responders based on tumour uptake. Furthermore, $\left[{ }^{18} \mathrm{~F}\right] \mathrm{AlF}-\mathrm{mNOTA}-\mathrm{GZP}$ showed positive associations with changes in tumour-associated lymphocytes expressing GZB, namely GZB+ CD8+ T cells, and decreases in suppressive F4/80+ cells. [ $\left.{ }^{18} \mathrm{~F}\right] \mathrm{AlF}-\mathrm{mNOTA}-\mathrm{GZP}$ tumour uptake was mediated by GZB expressing CD8+ cells and successfully stratifies therapy responders from non-responders, potentially acting as a non-invasive biomarker for ICIs and combination therapy evaluation in a clinical setting.
\end{abstract}

Keywords: granzyme B; PET imaging; immunotherapy; CpG-ODN; [ $\left.{ }^{18} \mathrm{~F}\right] \mathrm{AlF}-\mathrm{mNOTA}-\mathrm{GZP}$

\section{Introduction}

Immunotherapy has emerged as the fourth pillar of cancer treatment, along with chemotherapy, radiotherapy, and surgery. Immunotherapy exploits the host immune system, activating it to identify and destroy cancer cells [1]. Immune checkpoint inhibitors (ICIs) block the checkpoint receptors that tumours use to evade the immune system, dampening $\mathrm{T}$ cell activation. However, despite rapid advancements in immunotherapy, durable response rates to ICIs remain low, especially in colorectal cancers which are typically microsatellite stable [2]. In order to address this, combination clinical trials are ongoing to assess which adjuvants can enhance responsiveness by increasing tumour immunogenicity. 
CpG-oligodeoxynucleotides (CpG-ODN) are small synthetic DNA fragments containing unmethylated cysteine-guanosine motif dinucleotides that stimulate the innate and adaptive immune systems. These $\mathrm{CpG}-\mathrm{ODNs}$ can mimic the immunostimulatory activity of bacterial DNA, activating Toll-like receptor 9 (TLR9) [3-6] present on the endosomes of plasmacytoid dendritic cells ( $\mathrm{pDCs}$ ) and $\mathrm{B}$ cells [5]. This activation induces a range of responses, including IFN- $\alpha$ release from $\mathrm{pDCs}$, IFN- $\gamma$ from NK cells, and the promotion of the Thelper (Th)1 response [7]. Additionally, CpG-ODN can stimulate the production of interleukin-6 (IL6) and the proliferation of B cells, thereby upregulating co-stimulatory molecules on antigen-presenting cells (APCs) and stimulating myeloid lineage cells, such as macrophages, monocytes, and conventional dendritic cells [DCs] [8]. Because of these properties, $\mathrm{CpG}-\mathrm{ODN}$ has been explored as an adjuvant for immune modulators to treat solid cancers $[9,10]$. Profound anti-tumoural activity has been observed when CPG-ODN is administered locally into the tumour [9] and enhanced when combined with either immunotherapy or radiotherapy $[10,11]$, leading to their clinical assessment as in situ vaccines [11-16].

Non-invasive assessment of response to these novel combination therapies is complicated. Currently, there are a lack of specific biomarkers capable of providing a readout of in situ immune responses to different treatment strategies, complicating interpretation of clinical trials combining different treatment strategies, such as in situ vaccines and ICIs. Numerous studies have demonstrated that tumour-associated immune infiltrates are required for immune therapy efficacy, and numerous imaging biomarkers have been developed to quantify immune cell infiltrates, such as the T lymphocyte populations CD3 and CD8. However, these biomarkers provide little context and can be negatively affected by immune suppression. Granzyme B is a serine protease released by active NK cells and CD8+ cytotoxic T-cells along with the pore-forming protein perforin to induce apoptosis in tumours providing information on tumouricidal activity. We have previously characterised a PET imaging-based peptide biomarker targeting granzyme B $\left(\left[{ }^{18} \mathrm{~F}\right] \mathrm{AlF}-\mathrm{mNOTA}-\mathrm{GZP}\right)$ and demonstrated that it can successfully stratify tumours responding to combinations of immune therapeutics from non-responding tumours [17,18]. In the current study, we have evaluated whether $\left[{ }^{18} \mathrm{~F}\right] \mathrm{AlF}-\mathrm{mNOTA}-\mathrm{GZP}$, can also be used for the stratification of response to $\mathrm{CpG}-\mathrm{ODN}$ in situ vaccine therapy combined with the immune checkpoint inhibitor $\alpha \mathrm{PD} 1$ in a syngeneic mouse model of colon cancer. Furthermore, we explored whether different routes of administration of $\mathrm{CpG}-\mathrm{ODN}$ could influence different tumourassociated immune responses, correlating tumour uptake of $\left[{ }^{18} \mathrm{~F}\right] \mathrm{AlF}-\mathrm{mNOTA}-\mathrm{GZP}$ to differences in tumour-infiltrating immune cells using FACS.

\section{Materials and Methods}

\subsection{General Information}

All reagents were purchased from commercial suppliers and used without further purification. H-Asp (OtBu)-H NovaSyn TG resin $(0.21 \mathrm{mmol} / \mathrm{g}$ ) was obtained from Merck (Singapore). Fmoc-amino acids, HATU and HOAt, were obtained from Advanced Chemtech (Sabah, Malaysia). Fmoc-glutamic acid was t-butyl protected. (p-SCN-Bn)-NOTA was purchased from Macrocyclics (Plano, TX, USA). Sep-Pak ${ }^{\circledR}$ light $\left(46 \mathrm{mg}\right.$ ) Accell ${ }^{\mathrm{TM}}$ plus QMA carbonate cartridges and Sep-Pak ${ }^{\circledR} \mathrm{C} 18$ light cartridges were purchased from Waters Corporation (Milford, CT, USA). Saline solution $(0.9 \% w / v)$ was purchased from Braun Medical Industries (Singapore). All other chemicals and reagents were purchased from Sigma-Aldrich (Singapore), Fisher Scientific (Waltham, MA, USA) and Tokyo Chemical Industry (Chuo-ku, Tokyo, Japan). Carrier free aqueous $\left[{ }^{18} \mathrm{~F}\right]$ fluoride was produced via the $\left[{ }^{18} \mathrm{O}(\mathrm{p}, \mathrm{n})^{18} \mathrm{~F}\right]$ nuclear reaction (GE PETtrace 860 cyclotron, Boston, MA, USA). Quality control of radiolabelled tracer was performed on a UFLC Shimadzu HPLC (Kyoto, Japan) system equipped with a dual-wavelength UV detector and a PMT-radio detector (FlowRam, Lab Logic, Broomhill, Sheffield, UK). Radioactivity measurements were made with a CRC-55tPET dose calibrator (Capintec, Florham Park, NJ, USA). 


\subsection{Radiochemistry of $\left[{ }^{18} \mathrm{~F}\right] A l F-m N O T A-G Z P$}

The synthesis of NOTA- $\beta$-Ala-Gly-Gly-Ile-Glu-Phe-Asp-CHO (mNOTA-GZP) was prepared using standard Fmoc chemistry characterisation was done by using HPLC and mass spectroscopy as previously described [17]. Radiochemistry of $\left[{ }^{18} \mathrm{~F}\right]$ AlF-mNOTAGZP was prepared as previously described [18]. $\left[{ }^{18} \mathrm{~F}\right]$ AlF-mNOTA-GZP was prepared as a colourless solution $(10 \%$ ethanol in saline, $\mathrm{pH}=7.4)$ with a non-decay corrected radiochemical yield of $19-25 \%$ (reaction time $\sim 50 \mathrm{~min}$ ). The purity of the radiotracer was $>98 \%$, and molar activity was $45 \pm 20 \mathrm{GBq} / \mu \mathrm{mol}(n=6)$.

\subsection{CpG-Animal Model}

All animal procedures were carried out according to Institutional Animal Care and Use Committee Singapore (IACUC No. 181399) and NIH guidelines. Five- to seven-week-old female BALB/c mice were purchased from InVivos (Singapore). A CT-26 murine colon model was developed as described previously [18]. CT-26 cells $(0.2 \mathrm{M})$ were implanted subcutaneously (s.c) into the right upper limb of BALB/c mice. Once the tumour had grown approximately $100 \mathrm{~mm}^{3}$ (after 5 days of implantation). The animals were randomised into treatment groups, treated either with rat IgG2a isotype control antibody, CpG-ODN monotherapy (300 $\mu \mathrm{g}$ of CpG-ODN 1585 oligonucleotide (Integrated DNA technology) (Leuven, Belgium) delivered either via intratumoural (IT) or intraperitoneal (IP) route on days 5, 8 and 11), $\alpha$ PD1 monotherapy ( $\alpha$ PD1 mAb clone RMP1-14 (Bio-X Cell, Lebanon, NH, USA $10 \mathrm{mg} / \mathrm{kg}$, IP) on days 6, 9 and 12), or a combination therapy groups ( $\alpha$ PD1 \& CpGODN (IT) or $\alpha$ PD1 \& CpG-ODN (IP) as per the regimen described above (Supplementary Figure S1A). Animals were monitored for tumour growth using callipers on days 5, 8, 12, 15 and 20 after tumour implantation. Tumour volume was calculated using the modified ellipsoid formula 1/2(Length $\times$ Width $^{2}$ ) [19]. Tumour growth inhibition (\%TGI) was determined using the formula TGI $(\%)=(\mathrm{Vc}-\mathrm{Vt}) /(\mathrm{Vc}-\mathrm{Vo}) \times 100$, where $\mathrm{Vc}$ and $\mathrm{Vt}$ are the mean tumour volume of control and treated groups at the end of the study and Vo at the start.

\subsection{Small Animal PET-CT Imaging}

As previously described, small animal PET-CT imaging was performed 14 days after tumour inoculation using a Siemens Inveon PET-CT [18]. Mice were anaesthetised using isoflurane gas (maintained at $1.2 \%$ alveolar concentration) and injected with $\left[{ }^{18} \mathrm{~F}\right] \mathrm{AlF}-$ mNOTA-GZP ( 15 MBq) via the lateral tail vein. A 10-min static PET acquisition was performed at $60 \mathrm{~min}$ post-injection (p.i.) of the tracer, and a CT scan was acquired for anatomical co-registration. Animals were monitored during imaging using the BioVet physiosuite for their body temperature and respiration rate. The reconstruction and the data analysis were performed as described previously $[17,18]$ using Amide software (version 10.3 Sourceforge). The radiotracer uptake in tissues was determined and normalised to injected dose and converted to a percentage of injected dose per gram of tissue (\% ID/g).

\subsection{Fluorescence-Assisted Cell Sorting (FACS)}

Tumours were excised immediately after in vivo PET imaging and freshly processed for flow cytometry. A single-cell suspension was generated by incubating in modified RPMI (Gibco) supplemented with 10\% heat-inactivated fetal bovine serum (Gibco, Life Technologies, Waltham MA, USA), $20 \mu \mathrm{g} / \mathrm{mL}$ of DNAse1 (Sigma-Aldrich, Singapore) and $200 \mu \mathrm{g} / \mathrm{mL}$ of Collagenase (Sigma-Aldrich, Singapore). The samples were mechanically diced and incubated for $1 \mathrm{~h}$ at $37^{\circ} \mathrm{C}$ and dissociated into single cells by passing through a $100-\mu \mathrm{m}$ cell strainer. The samples were then counted and assessed for viability with Trypan Blue using hemocytometer (Sigma-Aldrich, Singapore). One million (1 M) viable cells from each sample were plated into a 96-well plate, and cells were stained for FACS analysis with different antibodies as per the manufacturer's instructions. We looked at cell proportions as a change in percentage of either the parent population, rather than absolute cell counts. The gating strategy for the FACS analysis was presented in Supplementary Figure S2, and 
the gating was optimised from the spleen and tumour samples treated form the control IgG mice. The cells were stained with antibodies against CD103 (clone M290 FITC; BD Biosciences, San Jose, CA, USA), CD25 (clone PC61 BB700; BD Biosciences), CD45 (clone 30F11 BUV395; BD Biosciences), Fixable Live/Dead Blue (Invitrogen), CD62L (clone MEL-14 BUV563; BD Biosciences), CD86 (clone GL1 BUV615; BD Biosciences), F4/80 (clone T452342 BUV661; BD Biosciences), NKp46 (clone 29A1.4 BUV737; BD Biosciences), CD3e (clone 500A2 BUV805; BD Biosciences), FoxP3 (clone 150D AlexaFluor647; Biolegend), CD44 (clone IM7 APC-R700; BD Biosciences), CD11b (clone M1/70 APC-Cy7; Biolegend, London, UK), granzyme B (clone QA16A02 PE; Biolegend), CCR7 (clone 4B12 PE-CF594; BD Biosciences), CD19 (clone 6D5 PE-Cy5; Biolegend), CD206 (clone C068C2 PE-Cy7; Biolegend), CD127 (clone SB/199 BV421; BD Biosciences), Ly6G (clone 1A8 BV480; BD Biosciences), CD8a (clone 53-6.7 BV510; BD Biosciences), CD11c (clone N418 BV570; Biolegend), Ly6C (clone HK1.4 BV605; Biolegend), Siglec F (clone E50-2440 BV650, BD Biosciences), CD68 (clone FA-11 BV711; Biolegend), CD4 (clone GK1.5 BV750; BD Biosciences) and I-A/I-E (clone M5/114.15.2 BV785; Biolegend).

Flow cytometry was performed on a BD FACSymphony. Fluorophore compensations and detector voltage were set up using single stains on murine spleen cells. Data was recompensated and analysed using FlowJo V10.7.1 software (FlowJo LLC, Ashland, OR, USA).

\subsection{Statistical Analysis}

All data were analysed using a 1-way ANOVA (Kruskal-Wallis) with a Dunn's posttest using GraphPad Prism (GraphPad Software v 8.0.0, San Diego, CA, USA). Statistical significance was considered where $p<0.05$. Data are expressed as mean \pm S.D. unless otherwise indicated.

\section{Results}

\subsection{Assessment of Treatment Efficacy Using Tumour Growth Volume}

Mice bearing colon CT-26 tumours were treated with control IgG, $\alpha$ PD1, CpG-ODN (IT), CpG-ODN (IP), or a combination of $\alpha$ PD1 + CpG-ODN (IP) or $\alpha$ PD1 + CpG-ODN (IT) (Supplementary Figure S1A) and tumour volumes measured over time. We observed that CT-26 tumour growth curves were normally distributed (Shapiro-Wilk $p>0.68$ ) (Supplementary Figure S1B) and exhibited different responses to monotherapy or combination therapies based on the tumour volume measurements (individual and grouped tumour volumes are shown in Figure 1A,B). Tumour volume changes indicate that local IT CpGODN delivery is required for anti-tumour effects and combined $\alpha$ PD1 + CpG-ODN (IT) elicited even greater anti-tumour activity (Figure 1C, Supplementary Table S1).
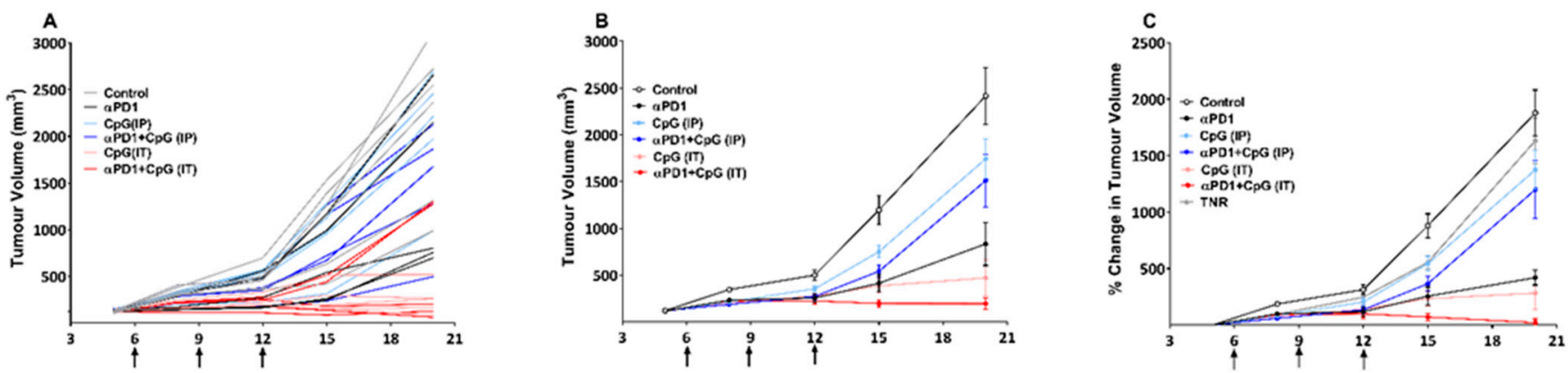

Figure 1. Assessment of the therapeutic effect of $\mathrm{CpG}-\mathrm{ODN}$ and $\alpha \mathrm{PD} 1$ combinations on changes in CT-26 tumour volume. (A) Individual animal tumour volume data measured on days 5, 9, 12, 15 and 20 post-implantation. (B) Group averaged tumour volume data from each treated cohort. (C) Percentage change in tumour volume after separation into TR and TNRs based on \%TGI. All data are shown as mean \pm S.D. (where TR, treated responder and TNR, treated non-responder). Arrow indicates the dosing of $\alpha \mathrm{PD}-1$. 
Tumour volumes were converted to percentage tumour growth inhibition (\%TGI), and $\left[{ }^{18} \mathrm{~F}\right]$ AlF-mNOTA-GZP tumour uptake was correlated across all data sets before post hoc manipulation (Pearson $\mathrm{r}=0.654,{ }^{* * * *} p<0.0001, n=60$ ). When individual treatment groups were assessed prior to stratification, overall \% TGI and tumour uptake of $\left[{ }^{18} \mathrm{~F}\right] \mathrm{AlF}-$ mNOTA-GZP were found to be positively correlated only in the CpG-ODN (IT)- $(\mathrm{r}=0.703$, $\left.{ }^{*} p<0.05 n=10\right)$ and $\alpha$ PD1 + CpG-ODN (IT)-treated groups $\left(\mathrm{r}=0.806,{ }^{* *} p<0.01, n=10\right)$. The $\alpha$ PD-1 monotherapy cohort did not reach significant difference $(r=0.720, p>0.05)$ due to the variability in tumour responses $(<40 \%$ of response rate). Similarly, the monotherapy CpG-ODN (IP)-treated cohort $(\mathrm{r}=0.507, p>0.05)$ and combination therapy cohort of $\alpha$ PD1 + CpG-ODN (IP) ( $r=0.257, p>0.05)$ were not well correlated, perhaps due to low tumour accumulation and rapid clearance of CpG-ODN from the body (Supplementary Table S2).

An effective therapy response in this pre-clinical model was estimated by comparing the tumour volumes between day 6 (baseline) and day 20 (post-therapy volume). We separated the treatment arms into two groups based on tumour volume data, a treatment responder (where we included a partial and complete responder, TR) and a treatment nonresponder (TNR). This approach has been used previously in order to assess imaging agents for their ability to stratify responders from non-responders; however, this approach may still be prone to bias [18,20-23]. Therefore, TRs were identified as final tumour volumes less than $750 \mathrm{~mm}^{3}$ and include tumours with stable or decreased volumes (tumour volumes are shown in Supplementary Table S1). This tumour volume was selected as this is more than 3 times the standard deviation from the mean tumour volume of the control group on day 20 , and using this approach, there is only a less than $1 \%$ chance for a TR to be incorrectly assigned. Treatment response varied across the treatment arms, with $\alpha$ PD1 + CpG-ODN (IT) combined treated group exhibiting the most significant response rate (Supplementary Table S2).

\subsection{Assessment of Treatment Efficacy Using $\left[{ }^{18}\right.$ F]AlF-mNOTA-GZP PET Imaging}

In-vivo PET imaging with $\left[{ }^{18} \mathrm{~F}\right] \mathrm{AlF}-\mathrm{mNOTA}-\mathrm{GZP}$ revealed that tumours could be visualised above background, and the radiotracer uptake was found to be heterogeneous across different treatment arms (Figure 2A). $\left[{ }^{18} \mathrm{~F}\right] \mathrm{AlF}-\mathrm{mNOTA}-\mathrm{GZP}$ was able to stratify responders from non-responders across different therapy arms (Table 1 and Figure 2B,C). In general, low $\left[{ }^{18} \mathrm{~F}\right] \mathrm{AlF}-\mathrm{mNOTA}-\mathrm{GZP}$ tumour uptake was observed in the control group $(0.20 \pm 0.06 \% \mathrm{ID} / \mathrm{g}, n=10)$ and TNRs $(0.20 \pm 0.03 \% \mathrm{ID} / \mathrm{g}, n=10)$. Significantly higher uptake was observed in all TRs from both monotherapy $\alpha \mathrm{PD} 1\left(0.42 \pm 0.10 \% \mathrm{ID} / \mathrm{g},{ }^{*} p<0.05\right.$, $n=7)$ and CpG-ODN (IT) $\left(0.82 \pm 0.21 \% \mathrm{ID} / \mathrm{g},{ }^{* * * *} p<0.0001, n=6\right)$ and combination therapy $\alpha \mathrm{PD} 1+\mathrm{CpG}-\mathrm{ODN}$ (IP) $\left(0.49 \pm 0.08 \% \mathrm{ID} / \mathrm{g},{ }^{* *} p<0.01, n=5\right)$ and $\alpha \mathrm{PD} 1+\mathrm{CpG}-$ ODN (IT) $\left(0.95 \pm 0.12 \%\right.$ ID $\left./ g,{ }^{* * * *} p<0.0001, n=6\right)$ cohorts (Figure $\left.2 \mathrm{~B}\right)$. Furthermore, no significant uptake was observed in the CpG-ODN (IP) alone group compared to the TNR group $(0.26 \pm 0.17$ vs. $0.20 \pm 0.03 \% \mathrm{ID} / \mathrm{g}, p=0.779, n=10)$.

Tracer is mostly excreted through the urinary system, and the uptake in kidneys was $1.85 \pm 0.1 \% \mathrm{ID} / \mathrm{g}$, with lower clearance through the hepatobiliary system (liver uptake $1.87 \pm 0.34 \% \mathrm{ID} / \mathrm{g}$ ). We observed some uptake in immune-related organs, particularly the spleen; however, no significant differences in spleen uptake were observed between responders and non-responders $(0.81 \pm 0.02 \% \mathrm{ID} / \mathrm{g}$ vs. $0.65 \pm 0.06 \% \mathrm{ID} / \mathrm{g}, p>0.05)$ (Supplementary Figures S3 and S4). 


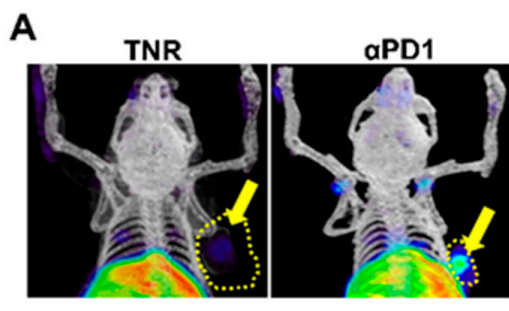

B

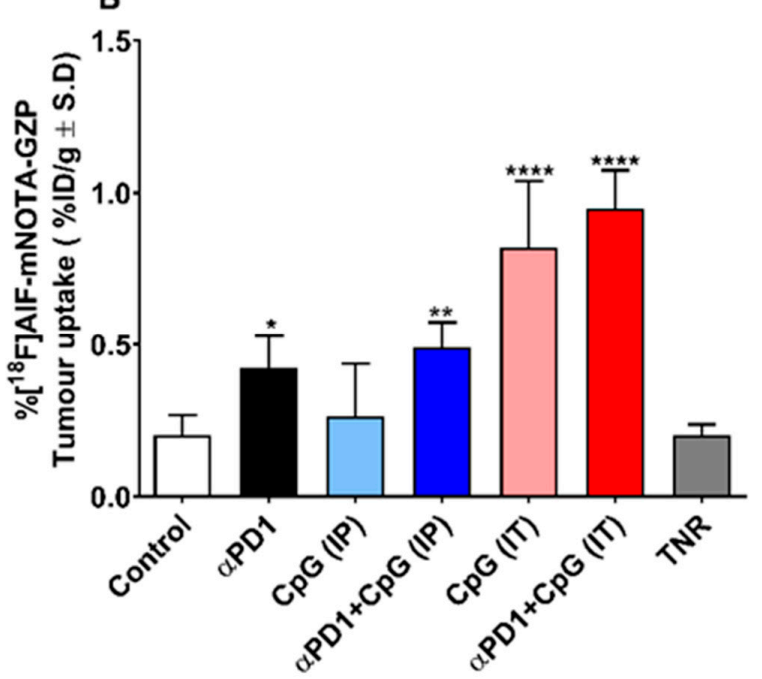

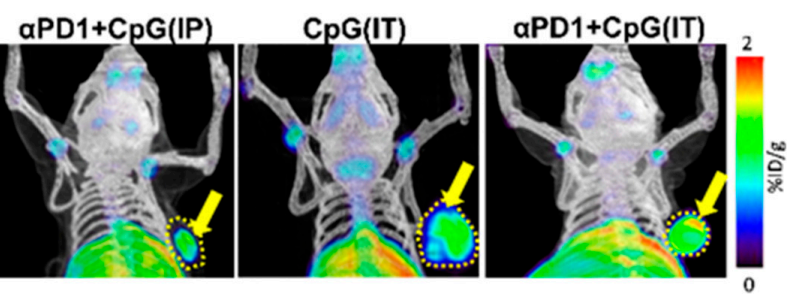

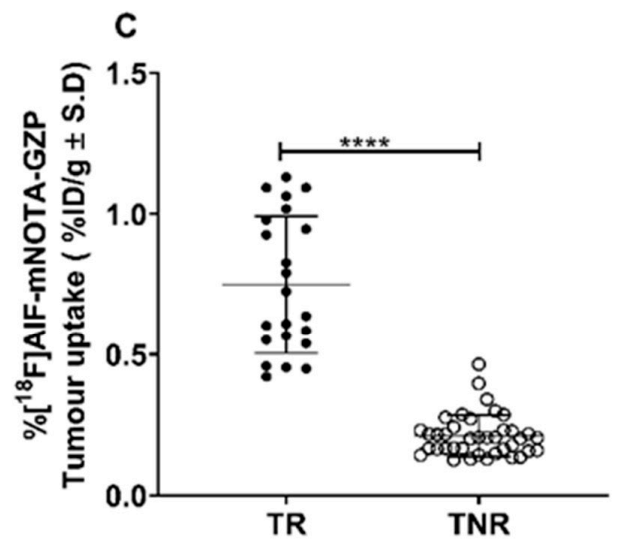

Figure 2. Fused PET/CT images of $\left[{ }^{18} \mathrm{~F}\right] \mathrm{AlF}-\mathrm{mNOTA}-\mathrm{GZP}$ from Balb/C mice. (A) MIP images showing $\left[{ }^{18} \mathrm{~F}\right] \mathrm{AlF}-\mathrm{mNOTA}-\mathrm{GZP}$ uptake in CT-26 tumours from treated non-responders (TNR), $\alpha \mathrm{PD} 1$ monotherapy, CpG(IP) monotherapy, CpG(IT) monotherapy, combined $\alpha$ PD1 + CpG (IP) and $\alpha$ PD1 + $\mathrm{CpG}$ (IT) treated responders. Yellow arrow indicates the position of the CT-26 tumour, and dashed line indicates tumour boundary. (B) Graph showing $\left[{ }^{18} \mathrm{~F}\right] \mathrm{AlF}-\mathrm{mNOTA}-\mathrm{GZP}$ uptake in responders from each treatment arm compared to TNR. Significant increases in $\left[{ }^{18} \mathrm{~F}\right]$ AlF-mNOTA-GZP tumour uptake were observed in $\alpha \mathrm{PD} 1\left({ }^{*} p<0.05, n=7\right)$ and CpG-ODN (IT) $(* * * * p<0.0001, n=6)$ monotherapy treated arms and $\alpha \mathrm{PD} 1+\mathrm{CpG}-\mathrm{ODN}(\mathrm{IP})\left({ }^{* *} p<0.01, n=5\right)$ and $\alpha \mathrm{PD} 1+\mathrm{CpG}-\mathrm{ODN}$ (IT) $\left({ }^{* * *} p<0.0001\right.$, $n=6)$ combination treated arms when compared to treated non-responders (TNR, $n=10$ ). No significant changes were observed in the CpG-ODN (IP) monotherapy group $(n=10)$ compared to TNRs. (C) Individual CT-26 tumour uptake of [ $\left.{ }^{18} \mathrm{~F}\right] \mathrm{AlF}-\mathrm{mNOTA}-\mathrm{GZP}$ in TR and TNRs overall $\left({ }^{* * * *} p<0.0001, n=39\right)$. Where, $\bullet$ Tumour responder (TR) and o Tumour non responder (TNR). The PET imaging was done 2 days after the last PD1 administration, and all data is provided as mean \pm S.D.

Table 1. Represents [ $\left.{ }^{18} \mathrm{~F}\right]$ AlF-mNOTA-GZP quantitative PET uptake in CT-26 tumour. ROIs were defined from individual CT-26 tumour-bearing mice subjected to different treatment cohorts, and the uptake values were converted to $\%$ ID/g. All data indicated as mean \pm S.D. of control groups, treatment responders (TR), and treatment non-responders (TNR) (where ${ }^{*} p<0.05 ;{ }^{* *} p<0.01$, **** $p<0.0001$ comparing T.R. to TNR).

\begin{tabular}{cc}
\hline Uptake of $\left[{ }^{18}\right.$ F]AlF-mNOTA-GZP in CT-26 Tumour \\
\hline Control & $0.20 \pm 0.06$ \\
Treatment Responder (TR) & $0.42 \pm 0.10^{*}$ \\
CpG-ODN (IP) & $0.26 \pm 0.17$ \\
$\alpha$ PD1 + CpG-ODN (IP) & $0.49 \pm 0.08^{* *}$ \\
CpG-ODN (IT) & $0.82 \pm 0.21^{* * *}$ \\
$\alpha$ PD1 + CpG-ODN (IT) & $0.95 \pm 0.12^{* * *}$ \\
Treatment Non-Responder (TNR) & $0.20 \pm 0.03$
\end{tabular}




\subsection{Immune Cell Profiling by FACS and Its Correlation to $\left[{ }^{18} \mathrm{~F}\right] A l F-m N O T A-G Z P$ Tumour Uptake}

FACS analysis was performed on day 14 after PET imaging in order to quantify the changes in immune infiltrates in response to therapy. Figure 3 demonstrates the immune cell composition of the CT-26 tumours and shows that overall response in this study is mainly mediated by GZB releasing T cells. A significant change was noticed in T cell populations, especially the $\mathrm{CD} 45+, \mathrm{CD} 8+$ and $\mathrm{CD} 4+\mathrm{T}$ cells. Separating them into different treatment cohorts revealed a significant increase in GZB+CD45+ T cells in all TRs from both monotherapy $\alpha \mathrm{PD} 1\left(36.40 \pm 3.26,{ }^{*} p<0.05\right), \mathrm{CpG}-\mathrm{ODN}$ (IT) $\left(38.41 \pm 3.11,{ }^{*} p<0.05\right)$, combination therapy $\alpha \mathrm{PD} 1+\mathrm{CpG}-\mathrm{ODN}(\mathrm{IP})(40.28 \pm 4.47, * p<0.05)$ and $\alpha \mathrm{PD} 1+\mathrm{CpG}-$ ODN (IT) $\left(43.12 \pm 3.48,{ }^{* *} p<0.01\right)$. However, there was no significant increase in the $\mathrm{GZB}+\mathrm{CD} 45+\mathrm{T}$ cell population in the CpG-ODN (IP) alone group when compared to TNRs $(27.46 \pm 3.74$ vs. $26.41 \pm 1.90, p>0.05)$ (Figure $3 \mathrm{~A}$ ).
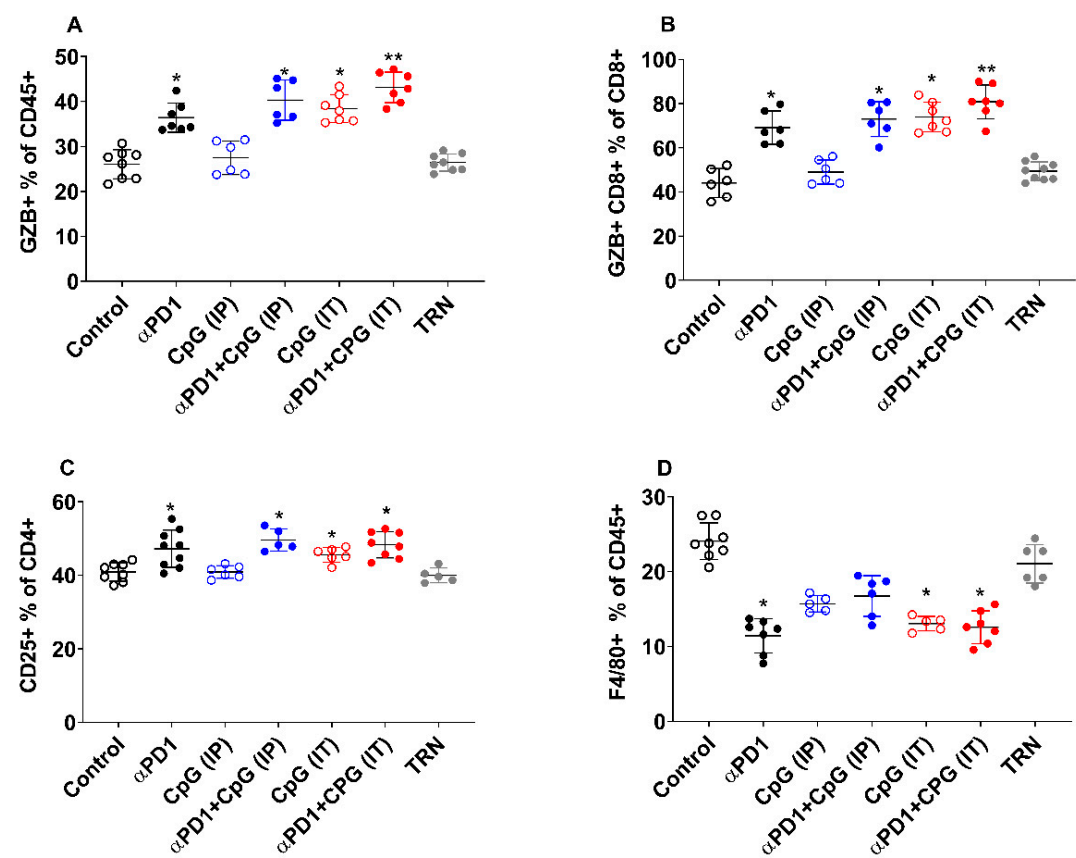

Figure 3. Multi-colour flow cytometry analysis of immune cells from mice bearing CT-26 tumour. Significant increases in T cell populations were observed, especially, the CD45+, CD8+ and CD4+ T cells. (A) Percentage of GZB+ cells relative to total CD45+ cells. A significant increase in GZB+CD45+ was noticed in all TRs from both monotherapy $\alpha \mathrm{PD} 1\left({ }^{*} p<0.05\right), \mathrm{CpG}-\mathrm{ODN}$ (IT) $\left({ }^{*} p<0.05\right)$, combination therapy $\left.\alpha \mathrm{PD} 1+\mathrm{CpG}-\mathrm{ODN}(\mathrm{IP}){ }^{*} p<0.05\right)$ and $\alpha \mathrm{PD} 1+\mathrm{CpG}-\mathrm{ODN}$ (IT) $\left({ }^{* *} p<0.01\right)$. However, no significant increase in the GZB+CD45+T cell population was noticed in the CpG-ODN (IP) monotherapy group $(p>0.05)$. (B) Percentage of GZB+ CD8+ cells relative to total CD8+ cells. Similarly, a significant infiltration of GZB+CD8+ cells was observed in $\alpha$ PD1 $(* p<0.05), \mathrm{CpG}-\mathrm{ODN}$ (IT) $(* p<0.05)$ and in combination therapy $\alpha \mathrm{PD} 1+\mathrm{CpG}-\mathrm{ODN}(\mathrm{IP})\left({ }^{*} p<0.05\right)$ and $\alpha \mathrm{PD} 1+\mathrm{CpG}-\mathrm{ODN}$ (IT) $\left.{ }^{* *} p<0.01\right)$. Again, no significant changes in GZB+CD8+ cell were observed in the CpG-ODN (IP) treated mice $(p>0.05)$. (C) CD25+CD4+ cells relative to total CD4+ cells, a significant increase in CD25+CD4+ cells in all TRs was noticed when compared to TNRs; $\alpha$ PD1 ( $\left.{ }^{*} p<0.05\right)$, CpG-ODN (IT) $\left({ }^{*} p<0.05\right)$ and combination therapy $\alpha \mathrm{PD} 1+\mathrm{CpG}-\mathrm{ODN}(\mathrm{IP})(* p<0.05)$, and the $\alpha \mathrm{PD} 1+\mathrm{CpG}-\mathrm{ODN}$ (IT) $\left.{ }^{*} p<0.05\right)$. CpG-ODN (IP) monotherapy group did not show any significant changes in the CD25+CD4+ cell population when compared to the TNR group (40.87 \pm 1.70 vs. $39.96 \pm 2.01$, $p>0.05)$. (D) Percentage of F4/80+ relative to total CD45+ cells across different treatment arms; $\alpha \mathrm{PD} 1$ monotherapy (* $\left.p<0.05), \mathrm{CpG-ODN}(\mathrm{IT}){ }^{*} p<0.05\right)$ and combination $\alpha \mathrm{PD} 1+\mathrm{CpG}-\mathrm{ODN}$ (IT) $\left.{ }^{*} p<0.05\right)$. However, no such reduction was observed in the CpG-ODN (IP) $(p>0.990)$ or combination $\alpha$ PD1 + CpG-ODN (IP) groups $(p>0.05)$ compared to TNRs. All data are represented as individual values with mean \pm S.D. $\left(n=5-10\right.$ mice/group. ${ }^{*} p<0.05 ;{ }^{* *} p<0.01$ compared to TNR). 
Similarly, a significant increase in the GZB+CD8+ cells was observed in monotherapy $\alpha \mathrm{PD} 1$ (69.16 $\left.\pm 7.55,{ }^{*} p<0.05\right), \mathrm{CpG}-\mathrm{ODN}$ (IT) $\left(73.87 \pm 6.70,{ }^{*} p<0.05\right)$ and in combination therapy $\alpha$ PD1 + CpG-ODN (IP) $(72.99 \pm 7.95, * p<0.05)$ and $\alpha$ PD1 + CpG-ODN (IT) $\left(80.83 \pm 7.62,{ }^{* *} p<0.01\right)$. Again, no significant changes were noticed in the GZB+CD8+ cells numbers in the CpG-ODN (IP)-treated mice in contrast to TNRs group (49.04 \pm 5.45 vs. $49.76 \pm 4.19, p>0.05$ ) (Figure $3 \mathrm{~B}$ ). In addition to CD8+ cells, we noticed a profound increase in the CD25+CD4+ cells in the monotherapy $\alpha \mathrm{PD} 1(47.20 \pm 5.05, * p<0.05), \mathrm{CpG}-\mathrm{ODN}$ (IT) $\left(45.52 \pm 2.00,{ }^{*} p<0.05\right)$ and combination therapy $\alpha$ PD1 + CpG-ODN (IP) $(49.60 \pm 2.99$, $\left.{ }^{*} p<0.05\right)$ and $\alpha \mathrm{PD} 1+\mathrm{CpG}-\mathrm{ODN}$ (IT) $\left(48.25 \pm 3.53,{ }^{*} p<0.0\right)$ responders. Again, the CpGODN (IP) monotherapy group did not show any significant changes in the CD25+CD4+ cell population when compared to the TNR group $(40.87 \pm 1.70$ vs. $39.96 \pm 2.01, p>0.05)$ (Figure 3C).

In addition, tumour responders not only increased the infiltration of GZB+ cells but at the same time, there was a concurrent significant reduction in tumour-associated suppressive cell types. F4/80+ macrophage reductions were observed in the treatment responding groups: $\alpha$ PD1 monotherapy $\left(11.44 \pm 2.29, p^{*}<0.05\right)$, CpG-ODN (IT) (13.05 \pm 0.977 , $\left.p^{*}<0.05\right)$ and combination $\alpha$ PD1 + CpG-ODN (IT) $\left(12.92 \pm 1.14, p^{*}<0.05\right)$. However, no such reduction was observed in the CpG-ODN (IP) $(15.69 \pm 1.08, p>0.990)$ or combination $\alpha \mathrm{PD} 1+\mathrm{CpG}-\mathrm{ODN}(\mathrm{IP})$ groups $(16.73 \pm 2.70, p>0.05)$ compared to TNRs $(21.03 \pm 2.56)$ (Figure 3D). Interestingly, no changes were observed in NK+ cells or GZB+ NK+ cell (Supplementary Table S3). Although there was a slight increase in the GZB+NKp46+ cells population in the TRs compared to the TNRs, this did not reach statistical significance $(16.01 \pm 1.91$ vs. $12.83 \pm 2.64, p>0.05)$. When looking at total lymphocyte cell composition as the \% GZB+CD45+, most of the cell type is associated with CD8+ and is significantly higher in TRs when compared to TNRs. We also noticed a majority of NK+ cells, CD4+ and other non-T cells at the tumour site (Supplementary Table S3).

\section{Discussion}

CpG-ODN is presented as an effective adjuvant to elicit a stronger anti-tumour response when combined with immune checkpoint inhibitors. CpG-ODN has been shown to modulate the immune system through engagement with TLR9 receptors expressed on plasmacytoid dendritic cells (pDC) or B cells $[6,24,25]$. The presence of unmethylated cysteine-guanosine motifs on CpG-ODN can mimic bacterial DNA and act as a pathogenassociated molecular pattern (PAMP) to trigger immune responses. To date, four classes of synthetic CpG-ODNs have been described, each with distinct structural and biological properties [26]. Type-A CpG-ODNs consist of mixtures of phosphorothioate/phosphodiester backbone along with a single motif flanked by palindromic sequences of poly $\mathrm{G}$ at $3^{\prime}$ and $5^{\prime}$ ends, which stimulate pDCs cells to secrete IFN- $\alpha$ and thereby strongly activate T cells. Type-B CpG-ODNs are encoded with multiple CpG on phosphorothioate backbone and act on pDCs to secrete TNF- $\alpha$, activating B cells to produce immunoglobulin M (IgM) as a first line of response to antigen exposure. Type-C CpG-ODNs combines features of both type-A and type-B, and can activate both T cells and B cells through IFN- $\alpha$ and IL-6 signaling, respectively. Type-P CpG-ODNs consist of two palindromic sequences of CpG motif, and act on both T cells and B cells; however, they secrete substantially higher amounts of IFN- $\alpha$ through pDCs cells when compared to type-C ODNs $[8,26]$. In this study, we have used CpG-ODN 1585, which belongs to type-A CpG-ODNs, which stimulate and activate T cells through engaging TLR9 expressed on the pDC cells.

CpG-ODN is being explored clinically for use as a vaccine adjuvant in combination with ICIs, chemo, or radiotherapy for various cancer types (metastatic prostate cancer, melanoma, or recurrent head and neck cancer) [25,27-29]. However, accurate assessment of immune treatment response in a clinical setting is complex [30,31]. Typically, radiological measures of tumour volume are employed to assess response to treatment in solid tumours; however, new response types associated with immune therapies, such as pseudoprogres- 
sion, hyperprogression, or a dissociative response may not be accurately interpreted with these conventional response criteria [32,33].

Non-invasive molecular imaging techniques, such as PET imaging, enable the visualisation and quantification of radiopharmaceutical uptake in the lesions, allowing for the assessment of changes in the tumour microenvironment in response to different cancer immunotherapies or combination therapies. Here, we have demonstrated the feasibility of $\left[{ }^{18} \mathrm{~F}\right] \mathrm{AlF}-\mathrm{mNOTA}-\mathrm{GZP}$, a probe binding to granzyme B released by activated cytotoxic $\mathrm{T}$ cells, to act as a surrogate biomarker of response to $\mathrm{CpG-ODN}$, alone or in combination with $\alpha$ PD1. CpG-ODN elicited a stronger anti-tumoural effect when administered intratumourally compared to intraperitoneally (Figure $1 \mathrm{C}$ ), a result mirrored by greater tumour retention of $\left[{ }^{18} \mathrm{~F}\right] \mathrm{AlF}-\mathrm{mNOTA}-\mathrm{GZP}$ (Figure $2 \mathrm{~B}$ ). Local administration of CpG-ODN creates a stronger inflammatory response at the tumour site, activating tumour-associated pDCs, secreting higher amounts of IFNs, which in turn activate T cells, dendritic cells and NK cells. In addition, local administration of CpG-ODN can significantly increase the production of pro-inflammatory chemokines and cytokines, such as IP10, MIP1 $\beta$, MCP5, MIP1, RANTES, JE, MCP5 and MIP1 $\alpha$, which play a role in the observed anti-tumour effects [9]. A previous study by Lou et al., demonstrated that systemic administration of CpG-ODN does produce an immune response; however, the activated immune cells fail to migrate to the tumour site [9]. Surprisingly, while the combination of $\alpha$ PD1 and CPG-ODN (when administered intratumorally) showed a small improvement in tumouricidal efficacy when measured tumour volume was measured, no significant potentiation of $\left[{ }^{18} \mathrm{~F}\right] \mathrm{AlF}-$ mNOTA-GZP tumour uptake was observed when compared to CpG-ODN when applied intratumorally alone. Despite this, $\left[{ }^{18} \mathrm{~F}\right] \mathrm{AlF}-\mathrm{mNOTA}-\mathrm{GZP}$ successfully stratified the responding tumours (TRs) from non-responders (TNRs). The increased tumour uptake of $\left[{ }^{18} \mathrm{~F}\right] \mathrm{AlF}-\mathrm{mNOTA}-\mathrm{GZP}$ in TRs was associated with a robust increase in tumour-associated activated T cells, especially GZB+ CD8+ cells, GZB+ CD45+ cells and CD25+ CD4 cells (Figure 3 ) are in line with previously reported data $[34,35]$. In tumour responders, we also found a substantial reduction in tumour-related F4/80+ macrophages, a cell type normally linked with an immune-suppressive microenvironment. However, further experiments will be required to fully understand the effects on different macrophage subpopulations. We had anticipated an increase in tumour-associated $\mathrm{NK}+$ cells following intratumoural administration of CpG-ODN due to its indirect effects on these cell subtypes. However, none of the treatments induced significant changes in $\mathrm{NK}+$ cell numbers.

Further work is needed to determine whether these data are clinically translatable. A simple amino acid substitution (IEFD to IEPD) confers selectivity for human granzyme B [22], potentially providing a platform for further development of granzyme B peptides as companion diagnostic able to simplify interpretation of clinical trials involving immune therapy combinations.

\section{Conclusions}

In summary, we have assessed the ability of $\left[{ }^{18} \mathrm{~F}\right] \mathrm{AlF}-\mathrm{mNOTA}-\mathrm{GZP}$ to stratify response to combined $\alpha$ PD1 therapy and in situ vaccination with CpG-ODN in a CT-26 murine colon cancer model. Therapy response was mediated by GZB expressing CD8+ cells and strongly correlated to [ ${ }^{18} \mathrm{~F}$ ]AlF-mNOTA-GZP tumour uptake. Potentially, granzyme B imaging agents could be developed as a biomarker for adjuvant in situ vaccine therapy for use in combination with ICIs in a clinical setting.

Supplementary Materials: The following are available online at https: / www.mdpi.com/article/ 10.3390 / pharmaceutics14010150/s1, Figure S1A. Graphical representation of initiation of the study followed by dosing, tumour measurement, PET imaging, and FACS. Balb/C mice $(n=10$ per group) bearing CT-26 tumour were treated with control IgG, $\alpha$ PD1, CpG-ODN(IP), CpG-ODN(IT), or combinations of $\alpha \mathrm{PD} 1+\mathrm{CpG}-\mathrm{ODN}(\mathrm{IP})$ or $\alpha \mathrm{PD} 1+\mathrm{CpG}-\mathrm{ODN}$ (IT). B. CT-26 tumour growth curves showed normal distribution (Shapiro-Wilk $\mathrm{p}$ 0.683) and a different response to monotherapy or combination therapy. Figure S2. Representative flow cytometry gating strategy of the single-cell dissociated tumour cells. Gating strategy highlights the populations with significant differences 
mentioned in the main text (red boxes). This gating strategy shows flow plots derived from a control IgG treated mouse. Figure S3. The PET-derived biodistribution of $\left[{ }^{18} \mathrm{~F}\right] \mathrm{AlF}-\mathrm{mNOTA}-\mathrm{GZP}$ uptake in selected organs from the treatment responder (TR) and treatment non-responder (TNR) groups. The ROI was manually delineated, and the tracer uptake was extracted, adjusted to the injected dosage, and converted to a percentage injected dose per gram of tissue (percent ID/g). All data is based on the average of five animals with SEM. Figure S4. A maximum intensity projection (MIP) PET/CT fused image of $\left[{ }^{18} \mathrm{~F}\right] \mathrm{AlF}-\mathrm{mNOTA}-\mathrm{GZP}$ from Balb/C mice bearing CT-26 tumour responder, treated with $\alpha \mathrm{PD} 1+\mathrm{CpG}$ (IT). Tracer is excreted mainly through urinary routes, as a strong uptake in the bladder and kidney. Furthermore, there was strong absorption in the liver and gallbladder, indicating that the tracer is excreted via the hepatobiliary pathway. There is less uptake in the bone, indicating that the tracer is stable in vivo, and uptake in the responder tumour is much stronger than in the nonresponder tumour. Where, T-Tumour, L-Liver, K-kidney, and B-bladder. Table S1: The table shows the summary of tumour volumes in controls, $\alpha$ PD1, CpG-ODN (IP), $\alpha$ PD1 + CpG (IP), CpG-ODN (IT), and $\alpha \mathrm{PD} 1+\mathrm{CpG}-\mathrm{ODN}$ (IT.) treatment responders (TRs) and treatment non-responders (TNRs) across all therapy arms in the syngeneic CT-26 colon cancer model. Table S2: The tumour volume data were converted to the percentage of tumour growth inhibition (\%TGI) by comparing the volumes between day 5 and day 20 for each treatment. Table S3. Table shows the tumour-associated immune cell populations from CT-26 tumour-bearing mice after 14 days of monotherapy $\alpha$ PD1, CpG-ODN (IP/IT) or combination therapies cohorts. Overall data presented in this study is mainly mediated by GZB-releasing T cells. Significant changes in T cell populations, especially, the CD45+, CD8+ and CD4+ T cells were observed. In addition, all treatment responders showed significant reductions in F4/80+ cells compared to TNRs and control. Data are shown as mean $\%$ of cells \pm S.D. and represent $n=5-10$ mice/group, ${ }^{*} p<0.05 ;{ }^{* *} p<0.01$, comparing TR to TNR.

Author Contributions: Conceptualization, S.V.H. and J.L.G.; methodology, S.V.H., B.R., T.Y.X., T.J.R., S.K., P.C. and Y.Y.H.; formal analysis, S.V.H., J.L.G. and Y.Y.H.; investigation, S.V.H., B.R., J.L.G. and Y.Y.H.; writing — original draft preparation, S.V.H. and J.L.G.; writing-review and editing, S.V.H., J.L.G., Y.Y.H. and E.G.R.; supervision, J.L.G.; project administration, S.V.H.; funding acquisition, J.L.G. and E.G.R. All authors have read and agreed to the published version of the manuscript.

Funding: This research was funded by Singapore's Health and Biomedical Sciences (HBMS) Industry Alignment Fund Pre-Positioning (IAF-PP) grant H18/01/a0/018, administered by the Agency for Science, Technology and Research (A*STAR).

Institutional Review Board Statement: All animal procedures were carried out according to Institutional Animal Care and Use Committee Singapore (IACUC No. 181399) and NIH guidelines.

Data Availability Statement: The data presented in this study are available in the article as well as in Supplementary Materials.

Acknowledgments: This work was supported by funding from the Agency for Science, Technology, and Research $\left(A^{*}\right.$ STAR), Institute of Bioengineering and Bioimaging (IBB), Singapore. The authors gratefully acknowledge the cyclotron radiochemistry team, especially David J. Green at the Clinical Imaging Research Centre (CIRC), National University of Singapore (NUS) for the provision of $\left[{ }^{18}\right.$ F $]$ fluoride.

Conflicts of Interest: The authors declare no conflict of interest.

\section{References}

1. McCune, J.S. Rapid Advances in Immunotherapy to Treat Cancer. Clin. Pharmacol. Ther. 2018, 103, 540-544. [CrossRef] [PubMed]

2. Fan, A.; Wang, B.; Wang, X.; Nie, Y.; Fan, D.; Zhao, X.; Lu, Y. Immunotherapy in colorectal cancer: Current achievements and future perspective. Int. J. Biol. Sci. 2021, 17, 3837-3849. [CrossRef] [PubMed]

3. Heckelsmiller, K.; Rall, K.; Beck, S.; Schlamp, A.; Seiderer, J.; Jahrsdörfer, B.; Krug, A.; Rothenfusser, S.; Endres, S.; Hartmann, G. Peritumoral CpG DNA Elicits a Coordinated Response of CD8 T Cells and Innate Effectors to Cure Established Tumors in a Murine Colon Carcinoma Model. J. Immunol. 2002, 169, 3892. [CrossRef] [PubMed]

4. Li, J.; Song, W.; Czerwinski, D.K.; Varghese, B.; Uematsu, S.; Akira, S.; Krieg, A.M.; Levy, R. Lymphoma Immunotherapy with CpG Oligodeoxynucleotides Requires TLR9 Either in the Host or in the Tumor Itself. J. Immunol. 2007, 179, 2493. [CrossRef] [PubMed]

5. Kaczanowska, S.; Joseph, A.M.; Davila, E. TLR agonists: Our best frenemy in cancer immunotherapy. J. Leukoc. Biol. 2013, 93, 847-863. [CrossRef] 
6. Hemmi, H.; Takeuchi, O.; Kawai, T.; Kaisho, T.; Sato, S.; Sanjo, H.; Matsumoto, M.; Hoshino, K.; Wagner, H.; Takeda, K.; et al. A Toll-like receptor recognizes bacterial DNA. Nature 2000, 408, 740-745. [CrossRef]

7. Marshall, J.D.; Heeke, D.S.; Abbate, C.; Yee, P.; Van Nest, G. Induction of interferon- $\gamma$ from natural killer cells by immunostimulatory CpG DNA is mediated through plasmacytoid-dendritic-cell-produced interferon- $\alpha$ and tumour necrosis factor- $\alpha$. Immunology 2006, 117, 38-46. [CrossRef]

8. Bode, C.; Zhao, G.; Steinhagen, F.; Kinjo, T.; Klinman, D.M. CpG DNA as a vaccine adjuvant. Expert Rev. Vaccines 2011, 10, 499-511. [CrossRef]

9. Lou, Y.; Liu, C.; Lizée, G.; Peng, W.; Xu, C.; Ye, Y.; Rabinovich, B.A.; Hailemichael, Y.; Gelbard, A.; Zhou, D.; et al. Antitumor activity mediated by CpG: The route of administration is critical. J. Immunother. 2011, 34, 279-288. [CrossRef]

10. Kell, S.A.; Kachura, M.A.; Renn, A.; Traquina, P.; Coffman, R.L.; Campbell, J.D. Preclinical development of the TLR9 agonist DV281 as an inhaled aerosolized immunotherapeutic for lung cancer: Pharmacological profile in mice, non-human primates, and human primary cells. Int. Immunopharmacol. 2019, 66, 296-308. [CrossRef]

11. Hammerich, L.; Binder, A.; Brody, J.D. In situ vaccination: Cancer immunotherapy both personalized and off-the-shelf. Mol. Oncol. 2015, 9, 1966-1981. [CrossRef]

12. Mason, K.; Hunter, N. CpG plus radiotherapy: A review of preclinical works leading to clinical trial. Front. Oncol. 2012, 2, 101. [CrossRef]

13. Frank, M.J.; Reagan, P.M.; Bartlett, N.L.; Gordon, L.I.; Friedberg, J.W.; Czerwinski, D.K.; Long, S.R.; Hoppe, R.T.; Janssen, R.; Candia, A.F.; et al. In Situ Vaccination with a TLR9 Agonist and Local Low-Dose Radiation Induces Systemic Responses in Untreated Indolent Lymphoma. Cancer Discov. 2018, 8, 1258-1269. [CrossRef]

14. Ribas, A.; Medina, T.; Kummar, S.; Amin, A.; Kalbasi, A.; Drabick, J.J.; Barve, M.; Daniels, G.A.; Wong, D.J.; Schmidt, E.V.; et al SD-101 in Combination with Pembrolizumab in Advanced Melanoma: Results of a Phase Ib, Multicenter Study. Cancer Discov. 2018, 8, 1250-1257. [CrossRef]

15. Gallotta, M.; Assi, H.; Degagné, É.; Kannan, S.K.; Coffman, R.L.; Guiducci, C. Inhaled TLR9 Agonist Renders Lung Tumors Permissive to PD-1 Blockade by Promoting Optimal CD4+ and CD8+ T cell Interplay. Cancer Res. 2018, 78, 4943-4956. [CrossRef] [PubMed]

16. Vanpouille-Box, C.; Pilones, K.A.; Wennerberg, E.; Formenti, S.C.; Demaria, S. In situ vaccination by radiotherapy to improve responses to anti-CTLA-4 treatment. Vaccine 2015, 33, 7415-7422. [CrossRef]

17. Goggi, J.L.; Hartimath, S.V.; Hwang, Y.; Tan, Y.X.; Khanapur, S.; Ramasamy, B.; Jiang, L.; Yong, F.F.; Cheng, P.; Tan, P.W.; et al Examining Immunotherapy Response Using Multiple Radiotracers. Mol. Imaging Biol. 2020, 22, 993-1002. [CrossRef] [PubMed]

18. Goggi, J.L.; Tan, Y.X.; Hartimath, S.V.; Jieu, B.; Hwang, Y.Y.; Jiang, L.; Boominathan, R.; Cheng, P.; Yuen, T.Y.; Chin, H.X.; et al. Granzyme B PET Imaging of Immune Checkpoint Inhibitor Combinations in Colon Cancer Phenotypes. Mol. Imaging Biol. 2020, 22, 1392-1402. [CrossRef] [PubMed]

19. Tomayko, M.M.; Reynolds, C.P. Determination of subcutaneous tumor size in athymic (nude) mice. Cancer Chemother. Pharmacol. 1989, 24, 148-154. [CrossRef] [PubMed]

20. Aslan, K.; Turco, V.; Blobner, J.; Sonner, J.K.; Liuzzi, A.R.; Núñez, N.G.; De Feo, D.; Kickingereder, P.; Fischer, M.; Green, E.; et al Heterogeneity of response to immune checkpoint blockade in hypermutated experimental gliomas. Nat. Commun. 2020, 11, 931. [CrossRef] [PubMed]

21. Jiang, X.; Dudzinski, S.; Beckermann, K.E.; Young, K.; McKinley, E.; McIntyre, J.O.; Rathmell, J.C.; Xu, J.; Gore, J.C. MRI of tumor $\mathrm{T}$ cell infiltration in response to checkpoint inhibitor therapy. J. Immunother. Cancer 2020, 8, e000328. [CrossRef] [PubMed]

22. Larimer, B.M.; Wehrenberg-Klee, E.; Dubois, F.; Mehta, A.; Kalomeris, T.; Flaherty, K.; Boland, G.; Mahmood, U. Granzyme B PET Imaging as a Predictive Biomarker of Immunotherapy Response. Cancer Res. 2017, 77, 2318-2327. [CrossRef]

23. Rashidian, M.; LaFleur, M.W.; Verschoor, V.L.; Dongre, A.; Zhang, Y.; Nguyen, T.H.; Kolifrath, S.; Aref, A.R.; Lau, C.J.; Paweletz, C.P.; et al. Immuno-PET identifies the myeloid compartment as a key contributor to the outcome of the antitumor response under PD-1 blockade. Proc. Natl. Acad. Sci. USA 2019, 116, 16971. [CrossRef] [PubMed]

24. Krieg, A.M. Therapeutic potential of Toll-like receptor 9 activation. Nat. Rev. Drug Discov. 2006, 5, 471-484. [CrossRef] [PubMed]

25. Link, B.K.; Ballas, Z.K.; Weisdorf, D.; Wooldridge, J.E.; Bossler, A.D.; Shannon, M.; Rasmussen, W.L.; Krieg, A.M.; Weiner, G.J. Oligodeoxynucleotide CpG 7909 Delivered as Intravenous Infusion Demonstrates Immunologic Modulation in Patients With Previously Treated Non-Hodgkin Lymphoma. J. Immunother. 2006, 29, 558-568. [CrossRef] [PubMed]

26. Marshall, J.D.; Fearon, K.; Abbate, C.; Subramanian, S.; Yee, P.; Gregorio, J.; Coffman, R.L.; Van Nest, G. Identification of a novel CpG DNA class and motif that optimally stimulate B cell and plasmacytoid dendritic cell functions. J. Leukoc. Biol. 2003, 73, 781-792. [CrossRef] [PubMed]

27. Milhem, M.M.; Long, G.V.; Hoimes, C.J.; Amin, A.; Lao, C.D.; Conry, R.M.; Hunt, J.; Daniels, G.A.; Almubarak, M.; Shaheen, M.F.; et al. Phase $1 \mathrm{~b} / 2$, open label, multicenter, study of the combination of SD-101 and pembrolizumab in patients with advanced melanoma who are naïve to anti-PD-1 therapy. J. Clin. Oncol. 2019, 37, 9534. [CrossRef]

28. Available online: https:/ / clinicaltrials.gov/ct2/show / NCT03007732 (accessed on 14 February 2020).

29. Available online: https:/ / clinicaltrials.gov/ct2/history/NCT02927964?V_1=View (accessed on 30 June 2020).

30. Neves, A.A.; Brindle, K.M. Assessing responses to cancer therapy using molecular imaging. Biochim. Biophys. Acta (BBA)-Rev. Cancer 2006, 1766, 242-261. [CrossRef] 
31. Wolchok, J.D.; Hoos, A.; Day, S.; Weber, J.S.; Hamid, O.; Lebbé, C.; Maio, M.; Binder, M.; Bohnsack, O.; Nichol, G.; et al. Guidelines for the Evaluation of Immune Therapy Activity in Solid Tumors: Immune-Related Response Criteria. Clin. Cancer Res. 2009, 15, 7412. [CrossRef]

32. Yirgin, I.K.; Erturk, S.M.; Dogan, I.; Vatansever, S. Are radiologists ready to evaluate true response to immunotherapy? Insights Imaging 2021, 12, 29. [CrossRef]

33. Hodi, F.S.; Hwu, W.-J.; Kefford, R.; Weber, J.S.; Daud, A.; Hamid, O.; Patnaik, A.; Ribas, A.; Robert, C.; Gangadhar, T.C.; et al. Evaluation of Immune-Related Response Criteria and RECIST v1.1 in Patients with Advanced Melanoma Treated With Pembrolizumab. J. Clin. Oncol. 2016, 34, 1510-1517. [CrossRef]

34. Saillard, M.; Cenerenti, M.; Romero, P.; Jandus, C. Impact of Immunotherapy on CD4 T Cell Phenotypes and Function in Cancer. Vaccines 2021, 9, 454. [CrossRef]

35. Alam, I.S.; Mayer, A.T.; Sagiv-Barfi, I.; Wang, K.; Vermesh, O.; Czerwinski, D.K.; Johnson, E.M.; James, M.L.; Levy, R.; Gambhir, S.S Imaging activated T cells predicts response to cancer vaccines. J. Clin. Investig. 2018, 128, 2569-2580. [CrossRef] [PubMed] 